\title{
Sciendo
}

\author{
BULGARIAN ACADEMY OF SCIENCES
}

CYBERNETICS AND INFORMATION TECHNOLOGIES • Volume 19, No 3

Sofia 2019 Print ISSN: 1311-9702; Online ISSN: 1314-4081

DOI: $10.2478 /$ cait-2019-0030

\section{Smart Compact Laser System for Animation Projections}

\author{
S. Ilchev ${ }^{1}$, D. Petkov ${ }^{2}$, R. Andreev ${ }^{1}$, Z. Ilcheva ${ }^{1}$ \\ ${ }^{1}$ Institute of Information and Communication Technologies, Bulgarian Academy of Sciences, 1113 Sofia, \\ Bulgaria \\ ${ }^{2}$ Delta Lasers Technology Ltd., Voyvodinovo, Bulgaria \\ E-mails:svetozar@ilchev.net sys@deltalasers.com rumen@isdip.bas.bgrzlat@isdip.bas.bg
}

\begin{abstract}
In this paper, we present the design of a compact laser system for animation projections both indoors and outdoors. Our focus is on the hardware and software aspects of the electronic control of the system from the design phase to the experimental tests and evaluations. The main purpose behind our development efforts is the creation of an affordable laser system for research, entertainment and marketing purposes using contemporary advances in electronics, software development, semiconductor laser diodes and optics. The system is "smart" in the sense that it connects to other devices and the Internet via a WiFi network, so that in addition to doing standalone laser projections, it also has remote control and remote debugging capabilities. Via its two embedded microcontrollers, the system offers an easy integration into existing Internet of Things (IoT) infrastructure. The experimental results are very promising and bring us closer to creating a viable product from both a technological and an economic standpoint.
\end{abstract}

Keywords: Laser system, Smart electronic control, Animation projection, Firmware, Multimedia embedded system, Internet of Things.

\section{Introduction}

Laser systems for multimedia applications such as indoor and outdoor musical events, opening ceremonies, celebrating public and private special events, etc., keep gaining more and more popularity. Their progress has been made possible by advances in the fields of semiconductor laser diodes, optics, electronics and the improvement of the necessary software programs and tools.

A typical laser system for multimedia purposes (Fig. 1) contains one or more semiconductor laser diodes that are mounted inside brass, copper or other heatconductive housings. The diodes and their housings are positioned on a metal plate (sometimes in a separate enclosure) in such a way that the laser beams coming from each laser diode can be combined by optical components such as dichroic prisms and mirrors. As a result, the beams are combined in a single beam capable of representing a good bright white color in the ideal case. 


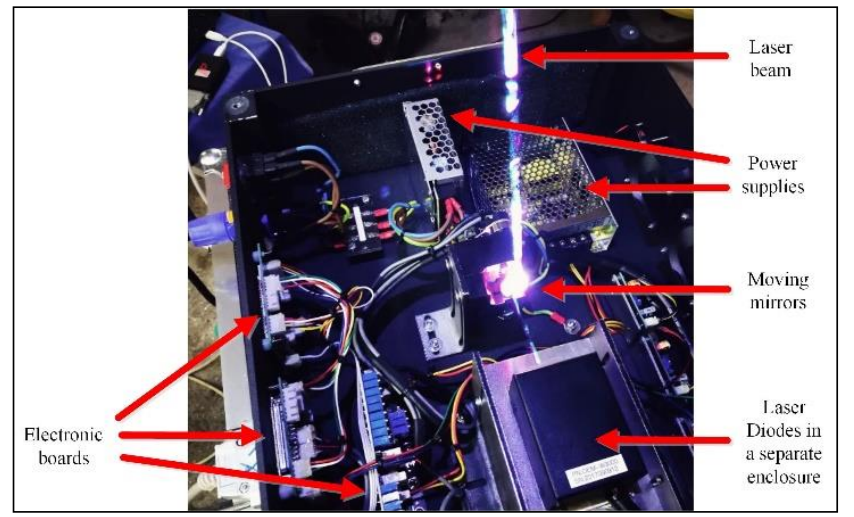

Fig. 1. Components of a typical laser system for multimedia purposes

At the next stage, the beam passes through two moving mirrors, which deflect it and project it onto a point on a surface in the distance. Each mirror is mounted on an axis and this axis is rotated by a galvanometer device controlled by an electronic board. The axes of the two mirrors are perpendicular to each other. In this way, a 2D area can be covered by the beam depending on the rotation angle of each mirror and an animation can be projected on the surface in the distance. The animation speed depends on the quality of the galvanometer units (also called scanners) with typical projection speeds ranging from 20 kilo-points per second (kpps) to $40 \mathrm{kpps}$. Usually, the scanners are controlled by a dedicated electronic board.

Each laser diode group - red, green or blue - is controlled by a separate electronic board called a Laser Diode (LD) driver. It contains a voltage-modulated analog or digital signal input and translates this input into the current that drives the corresponding laser diode group. The laser diode drivers and the scanner board are connected to a central ILDA board capable of receiving and interpreting analog signals according to the ILDA Standard Projector (ISP) specification [1] and/or digital signals most often transmitted through an Ethernet LAN connection.

As laser diodes generate a significant amount of heat, sometimes further cooling components are needed - Peltier elements (thermoelectric cooling) and fans. The ThermoElectric Cooling (TEC) has the advantage of pumping the heat very fast from the laser diodes and passing it to the large metal plates and the metal housing. The fans create sufficient airflow for taking the heat outside of the laser system. The cooling subsystem needs its own electronic control.

Such a laser system typically operates with a maximum optical output power ranging from several watts up to tens of watts. This amount of output power constitutes a significant danger to the human eye and special precautions are taken to ensure safe operation. Usually, a special key is needed to activate the laser system ensuring that only authorized personnel can turn it on. There is a safety switch to turn the laser beam off in cases of emergency and a physical shutter is often used to block the laser beam physically from exiting the laser system.

A typical laser system contains additional components such as power supplies, a control panel, air-filters for dust, etc. The control panel is typically mounted on the side of the system housing and it is connected to the central board. Besides the typical 
25-pin D-Sub input and output connectors for ILDA analog signals, the control panel may also contain a digital signal connector (e.g., RJ-45). Additional items on the panel may be potentiometers for setting the beam intensities of each color channel, key-switches for flipping the animations in both directions, a display for showing laser diode temperatures and other working parameters, an SD-card for storing laser animations, etc.

Laser systems capable of projecting animations are powerful but they are relatively big (e.g., a cubic shape with a side length between 50 and $75 \mathrm{~cm}$ ), heavy (usually above $10 \mathrm{~kg}$ ), expensive (several thousand dollars), often not waterproof and usually require expensive software support. For some applications - e.g., showing outdoor advertisements, projecting technical information in production facilities, displaying public information for citizens at train stations, etc., these systems are inadequate. They are unnecessarily powerful and complex, inconvenient to install, prohibitively expensive to purchase and operate, and they are prone to humidity damage in outdoor conditions.

There is a need for a compact, lightweight, cheap and waterproof laser system for undemanding applications targeting the public sector, marketing companies and production industries. Target users usually have no experience running laser systems, so the system should be robust and simple to run without the need for complicated laser show software. It should be designed to operate in outdoor conditions with the capability of both standalone laser animation projection and remote control in an IoTfashion. This system will fill a market niche, which begins to form alongside the progress of semiconductor laser diodes and the increasing popularity of laser light sources for attracting human attention and constructing laser beam drawings even in relatively bright environments.

In this paper, we propose the design of such a system with a focus on its electronic control. The next section outlines some related work. Then, we illustrate the requirements we have identified in more detail along with our main design decisions. After that, we present a technical overview of the laser system along with its main hardware components, construction and assembly. After the overview, we discuss the creation of the firmware from a software point of view and elaborate on some challenges that we encountered. Then, we present the initial experimental results from our prototype system and conclude the paper with some ideas pertaining to our future work.

\section{Related works}

There are a number of publications and patents in the field of laser image and animation generation. In [2], authors research the use of new semiconductor laser sources for the purpose of achieving better image quality during projection. The new laser diodes offer high power with the possibility of achieving high speeds making them perfect for projection applications. In [3], authors from Robert Bosch have been granted a patent for a laser module containing several laser diodes with different wavelengths. It is aimed at portable image projectors in interactive multimedia applications. The authors of the US patent [4] propose a new design of an efficient 
laser diode driver. This design uses modern electronic components to reduce the wasted energy from the power supply when the laser diodes emit light.

Author of [5] proposes an increase in the brightness and power output of laser diodes by means of polarization. Two polarization states are used and for each polarization state, the light emitted by multiple laser diodes is combined into a single beam. Then, the two beams corresponding to the two polarization states are merged together. In [6], authors propose using laser sources with multiple wavelengths for imaging systems targeting and enabling image capturing with excellent resolution. Authors of [7] propose another innovative application of laser light sources - the concentrated energy transmission of several watts of power at distances of about ten meters for the purposes of supplying portable devices with power in a wireless manner. Such innovative use cases may bring additional value to a compact laser system like the one that we propose in this paper.

In [8], authors propose an intelligent industrial fiber laser with high-power output connected to a smart cloud. The industrial laser is operated as an "Internet-ofThings" device using data gathered from other similar lasers connected to the same cloud. Authors of [9] present a new approach for the development of a laser-based lighting system. The main purpose is to achieve better efficiency and quality of the light than in LED-based systems. Similar ideas about using laser diodes as light sources in illumination systems are discussed in [10]. Among the advantages are the absence of phosphor-based materials, the high power efficiency and the good color rendering obtained by mixing red, green, blue and amber laser light sources.

The authors of [11] propose an image projection device consisting of a laser light source, an optical fiber and a high-speed optical scanner that can project a lasergenerated image onto a surface near the end of the optical fiber. The small laser beam diameter permits the generation of a high-resolution image. In [12], the authors discuss the improvements in the lifetime and power efficiency of some blue and green laser diodes. For blue diodes, the lifespan is estimated to be in the order of 40,000 working hours at operating temperatures of $40{ }^{\circ} \mathrm{C}$. The authors of [13] discuss the power efficiency of laser diodes for projection purposes, e.g., $50 \mathrm{~mW}$ per color channel are enough to result in light with intensity corresponding to $20 \mathrm{~lm}$. Authors propose optimized blue and red laser diodes to increase the power conversion efficiency.

\section{Compact laser system - requirements and design}

The design of the proposed multi-color laser system is based on requirements that we identified after talking to potential users. In Fig. 2, we summarize these requirements in the Goal-oriented Requirements Language (GRL) notation [14, 15]. The overall requirements are represented as soft-goals that are not strictly independent of one another.

One of the major topics that constantly comes up in our talks with potential customers is related to the cost of our system, which should be as low as possible. From an economic standpoint, achieving low cost is a decisive advantage that very strongly influences the customer's decision to use laser systems in his or her 
economic activities. This led us to the design decision to simplify the system and use a standard video camera enclosure that is already available on the market. We also reduced the number of laser diodes to one laser diode per channel, which directly reduces the manufacturing costs. We have also made the conscious design decision to use open-source design and programming tools whenever possible, which lowers the investment expenditures significantly as commercial tools for the development of embedded systems can be quite expensive. It also ensures the long-term availability of our development environments and enables us to scale our development efforts quickly.

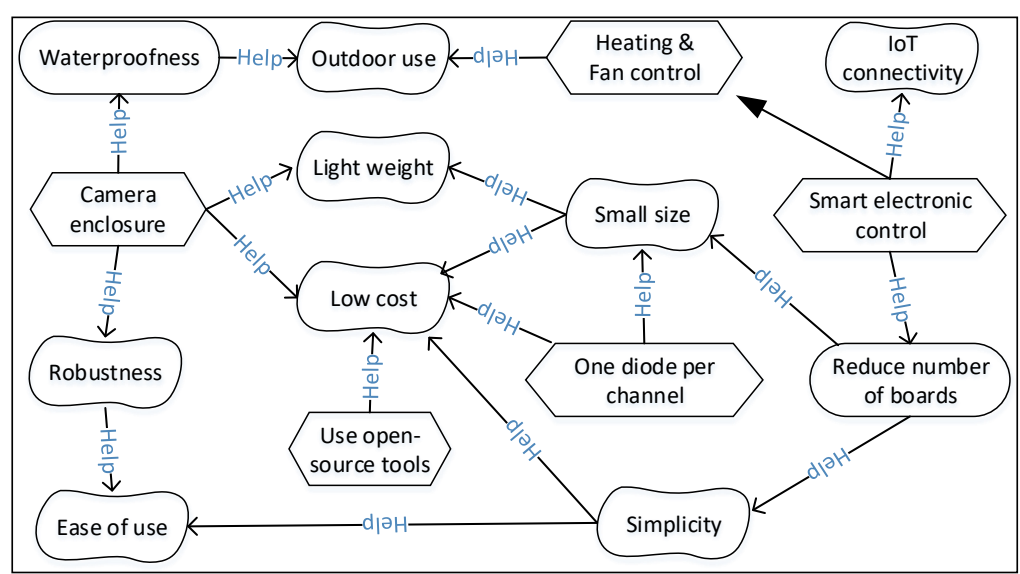

Fig. 2. Requirements in the Goal-oriented Requirements Language notation

Two important soft-goals that contribute to the low cost are the small size and the simplicity of the system. The small size is asked for by some of our customers, who want to mount the system in tight - most often very narrow - spaces. The simplicity of the system reduces our assembly and maintenance costs. We reduce the size by using fewer diodes and diode housings and by reducing the number of electronic boards in the system. The latter also contributes to the soft-goal of simplicity, as fewer boards are easier to install and setup.

The small size and the standard camera enclosure help us reduce the weight of the laser system. Customers, who transport the system often or mount it as part of a laser farm, often ask for lightweight systems that facilitate the physical handling so we have identified the reduction of weight as another soft-goal.

Customers also want a system that is easy to use. A simple and straightforward design and a robust system that can be relied upon contribute to this soft-goal. The premade camera enclosure contributes to the robustness and, in addition, makes our system waterproof. The waterproofness together with the heating and fan control are important prerequisites for the outdoor use - another one of our soft-goals. Some customers have asked us for a system that can be used outdoors to project laser animations to attract attention or for marketing purposes. Thus, the heating and fan control has become an important design feature implemented by our smart electronic control board. 
We use the term "smart" to denote, on one hand, the capability of the board for standalone operation and laser animation projection and, on the other hand, its capability to connect to the Internet, which enables the integration of the laser system into different kinds of IoT infrastructures. This kind of IoT connectivity is another important soft-goal, which gives us the option to monitor and control the laser system and its animation projections remotely. The number and range of clients that are interested in this kind of control and integration keeps increasing steadily, e.g., factory managers, shop owners, laser show organizers, etc.

The requirements presented above lead to some changes in the physical form of the system and its components, which we present from a technical point of view and illustrate with the aid of Fig. 3. The structure of the smart compact laser system is shown in Fig. 3 (right). It is adapted from the structure of a generic laser system (Fig. 3 , left) to reflect our needs (represented as soft-goals in Fig. 2) for simplification, compact dimensions, lower weight, lower cost, robustness, outdoor operation, ease of use and IoT connectivity.
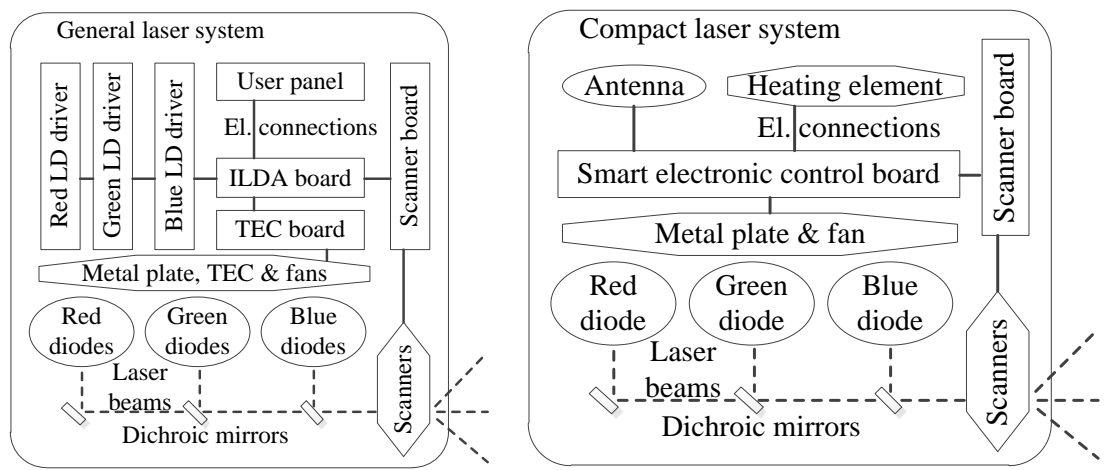

Fig. 3. Components of a generic laser system (left) and the smart compact laser system (right)

Instead of laser diode strings, in our compact system, we use only one laser diode per color channel. This generates less heat and makes possible the reduction of the metal plate size and the removal of the TEC and its electronic control board. A single fan is used to pump out the generated heat and balance the humidity.

We reduce the six boards to only two boards. The scanner board is bought together with the scanner units and is used as a separate board. The three LD drivers are simplified and housed together on the smart electronic control board. The ILDA board is removed as we do not envision any wired connectivity for signal transmissions to the system. The functions of the user panel (e.g., switches, display, etc.) are not implemented in a physical form. Instead, remote control and monitoring features are offered through the WiFi capability of the smart electronic control. For this purpose, the system has an optional dedicated WiFi antenna.

Besides the optional antenna, the other new element in the system is the heating element. As the system targets outdoor use-cases, it needs to consider some environmental parameters, the most important of which are the temperature and the humidity. If the operating temperature inside the enclosure is negative, the heating element is switched on to raise it above $0{ }^{\circ} \mathrm{C}$ as laser diodes cannot operate otherwise. 
If the temperature is too high, the fan is activated. The humidity also needs to be within an acceptable range to avoid condensation issues. The smart electronic control is provisioned with two sensors for temperature and humidity measurement, and the heating element and the fan are actively controlled according to their values.

\section{Compact laser system - technical overview}

In the technical development of the compact laser system, we made use of our previous experience in the creation of laser diode drivers, e.g., [16], laser systems [17] and our expertise in network communications employed in embedded systems $[18,19]$. After identifying the requirements for our system, in this section we describe in more detail the mechanical, electrical and software aspects of our design. All components of the compact laser system are mounted inside a professional security camera enclosure that is already available on the market (Fig. 4, left). The camera enclosure is waterproof and it has two nozzles at the lower back side for power supply and communication cables. It is both cheaper and lighter than the usual cubic-shaped enclosures of generic laser systems and it can be mounted in a standardized way to a wall or a ceiling.

At the front of the enclosure, the scanners that deflect the laser beam are mounted on a metal plate fixed to the enclosure. The mirrors are mounted on galvanometer axes controlled by the green board mounted vertically at the back of the enclosure. The ten blue trimmers visible on this green board are used to configure scanner parameters such as the maximum animation scale in both directions. The laser beams (blue and green sources are visible in Fig. 4, left) are combined by a beam splitter and reflected by a stationary mirror (located at the bottom left corner of the enclosure) before they reach the two deflecting mirrors. Near the middle of the enclosure, the small cubic-shaped housings of the laser diodes can be seen. The red printed circuit board (PCB) mounted horizontally is the smart electronic control. It is designed and developed by us from scratch to conform to the requirements we identified in the previous section. Besides the components inside the enclosure, the compact laser system needs a power supply for the laser diodes and the smart electronic control (9-24V DC) and a split power supply for the scanner units. Both power supplies are mounted externally for the moment.

The PCB of the smart electronic control is shown in more detail in Fig. 4 (right). The main microcontroller unit (MCU) is a 32-bit ARM Cortex-M4, model STM32F303RDT6 of the F3 series, working at $72 \mathrm{MHz}$. It successfully processes laser animations at 25 frames per $1 \mathrm{~s}$ and it has sufficient resources for performing other tasks such as reading from the memory card, receiving remote control commands, measuring the working temperature, etc. An additional module housing an ESP8266 MCU is mounted on the right edge of the board in Fig. 6. It is responsible for the connectivity to WiFi networks and the Internet. This second MCU takes care of network-related communication tasks. It is connected to the main MCU via a standard Serial Peripheral Interface (SPI).

The board also has a slot for a microSD memory card whose main purpose is to store laser animations and configuration settings. The microSD card is connected to 
the MCU via another SPI interface. The SPI connection mode is license-free and allows the simple and legally unproblematic use of the storage media.
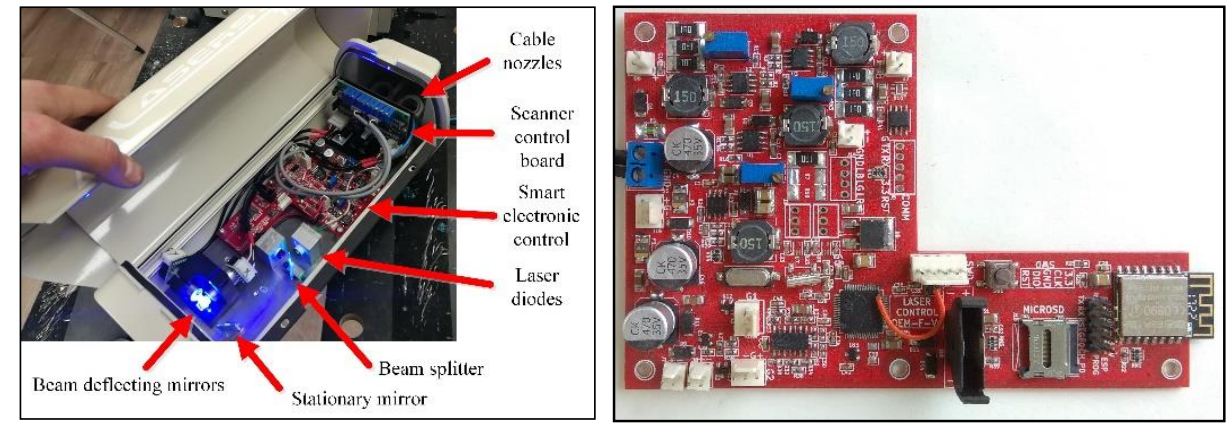

Fig. 4. The smart compact laser system (left) and the smart electronic control board (right)

The vertical socket for a CR2032 battery is used for real-time clock backup, so that if the main power supply is removed, the real-time clock is preserved and keeps updating. The programming and debugging is done via the Serial Wire Debug (SWD) interface. There are also electronics for controlling the scanner units - a quad operational amplifier, e.g., OPA1679IDR or OPA4171AIDR by Texas Instruments, and the corresponding resistors and capacitors. Both power supplies go through onboard fuses, ferrite filters and capacitors. There is a buck converter and a linear regulator for supplying respectively $5 \mathrm{~V}$ and $3.3 \mathrm{~V}$ to both MCUs and the other lowvoltage components.

At the upper part of the board, three very compact laser diode drivers are located. They are specialized buck converters designed to drive laser diodes (or diode strings) for the red $(\sim 638 \mathrm{~nm})$, green $(\sim 520 \mathrm{~nm})$ and blue $(\sim 450 \mathrm{~nm})$ color channels. For each color channel, the maximum operating current is in the range of $60 \mathrm{~mA}$ to $2.4 \mathrm{~A}$. The magnitude of the current range - milliamperes or amperes - is selected at the time of the board assembly by choosing suitable values for the nine current sensing resistors (three resistors per color channel). The milliampere range needs $10 \Omega$-resistors while the ampere range requires $1 \Omega$-resistors. The exact value of the current within the selected current range is set via an onboard trimmer separately for each driver. Depending on the forward voltage of the laser diodes, the operating current range translates to an electrical power consumption that ranges from $100 \mathrm{~mW}$ to $10 \mathrm{~W}$ per channel. The optical power is much less - ranging from $20 \mathrm{~mW}$ to $2 \mathrm{~W}$. The color intensity can be further controlled by the MCU while projecting the animation.

The PCB is fixed to the enclosure by means of five M3 screws. The antenna of the WiFi module is situated close to the front aperture to achieve better signal reception. There is also an option for mounting an external antenna if necessary.

The smart electronic control board has been devised and designed by us from scratch using the open-source eCAD software KiCad. The two-layer design ensures the unproblematic production and testing of the board on a hardware level. The board has protection mechanisms against accidental voltage polarity reversal, overvoltage and overcurrent conditions and overheating. 


\section{Electronic control - firmware design and challenges}

During the design and implementation phase, we identified several important activities that the software part - the firmware - of our smart compact laser system needs to take care of. These activities are performed concurrently in real-time and each of them requires different hardware and software capabilities.

The most important and time sensitive activity is the control of the scanners and the beam intensities of the laser diode drivers. Another real-time activity is the loading of the animation frames from the microSD card into the STM32 MCU memory. The electronic control also has to receive remote commands from the Internet, send status updates and manage network connections - an activity that is carried out in conjunction with the ESP8266 MCU. Last but not least, the temperature and the humidity inside the housing have to be managed and kept at acceptable values.

This multitude of activities necessitates the use of a Real-Time Operating System (RTOS). The RTOS we chose to use in our firmware is the open source FreeRTOS, which is a popular, reliable and efficient RTOS compatible with the STM32 series. The RTOS gives us the possibility to program each activity mentioned above as a separate program task. A program task is a piece of program code executed for a certain time period before the RTOS stops its execution and starts the execution of the next task. The length of the time slots allotted to the tasks can be configured along with a priority value for each task.

Our electronic control has a high-priority task for running the animation and three normal-priority tasks for reading data from the microSD card, communicating with the outside world and controlling the environmental parameters. The tasks are created statically after the initial setup of the laser system hardware and remain active throughout the lifetime of the firmware execution. The FreeRTOS scheduler is preemptive so that each task gets an execution time slot on a regular basis without the need for a task to yield the execution control explicitly. This makes the development easier and makes the program a little more robust against problems like unresponsiveness, starvation or deadlock.

The high-priority task for running the animation constantly changes the analog values controlling the scanners in each direction as well as the laser beam intensities for the red, green and blue color channels in order to achieve smooth animation projection. The values used in this process are read from a double buffer in the main MCU memory. This double buffer consists of two equally large memory sections. Each section holds the representation of an animation frame consisting of up to 1500 points. One frame is actively used by this task while the other one is updated by the normal-priority task responsible for reading data from the microSD card. The animation speed in frames/s can be set as a parameter, e.g., 25 frames per $1 \mathrm{~s}$.

The scanners are controlled using the Digital-to-Analog Converter (DAC) peripheral unit of the STM32 MCU. It has two channels with sufficient speed for our application. The DAC voltage output level ranges from $0 \mathrm{~V}$ to $3.3 \mathrm{~V}$. It has to be shifted to the standard input bipolar value range of $-10 \mathrm{~V}$ to $+10 \mathrm{~V}$ defined by the ILDA ISP standard [1]. This is done by means of the split power supply and the dedicated operational amplifier. The laser beam intensities for each channel are 
controlled by Pulse-Width Modulation (PWM). This is a convenient solution because no intermediate DAC units are needed and the intensity control can be done using a pair of integrated peripheral timer units on the STM32 MCU. Each timer unit has two PWM output channels that can be configured separately. After the configuration, the timer unit does the PWM generation automatically depending on the value of the capture/compare register for the specific output channel. In order to change the laser beam intensity, the MCU needs to change the PWM duty cycle by writing a new value into the corresponding capture/compare register. The timer unit does the actual change of the PWM without the further involvement of the main MCU. The PWM control also simplifies the laser diode driver units and paves the way to making them very compact in size so that all three driver units fit on the smart electronic control board.

The normal-priority task for reading data from the microSD card uses the open source library FatFs to read animation and configuration files saved on the FAT32 file system of the card. The animation files are read into the double buffer mentioned in the previous paragraph. The supported file format is the ILDA Image Data Transfer Format, last updated in 2014 [20]. It is a simple file format created for the storage of laser animations and it can be efficiently handled by embedded MCU units like the STM32. Unfortunately, it does not contain all parameters needed to project laser animations successfully on an arbitrary laser system because some projection parameters depend on the system itself. In some cases, additional metadata, e.g., for changing the blanking time of the laser beam may be necessary.

The ILDA file format consists of multiple sections ordered one after the other. Each section represents either an animation frame or a color palette. Right now, we support only frame sections and use for them the standard default ILDA color palette [20]. Each frame section contains a small 32-byte header followed by the data records that make up the animation frame. The header contains, among other information, the total number of data records for the frame. Each data record represents a laser beam point in the frame. It contains offsets in the $X$, the $Y$ and optionally the $Z$ directions for the laser scanners, a color index and a status code byte, which denotes whether the laser beam is on or off (laser beam blanking). This very simple structure of the ILDA file results in low overhead and allows each frame to be read directly into the buffer in the MCU memory with as little delay as possible. The reading procedure is as follows:

1. Determine the inactive memory slot in the double buffer;

2. Read the next frame header from the file into the memory slot and determine the total number of records;

3. Read all records for the next frame from the file.

Done in this style, the reading procedure is easily programmed to utilize the Direct Memory Access (DMA) peripheral unit of the MCU. The communication with the microSD card is implemented via an SPI port used as a source of data for the DMA unit. The DMA data destination is the inactive memory slot in the double buffer. Using the DMA unit frees up more MCU resources for processing the other application tasks. As the MCU memory size is usually not sufficient to hold the whole animation into the memory, the animation frames have to be constantly read from the 
microSD card into the buffer. The total number of frames per animation file is practically limited only by the 2-byte "Total frames" header field in the ILDA image format $(65,535$ animation frames per animation file) [20]. The total number of animation files depends practically on the microSD card size.

The normal-priority task for communicating with the Internet relies on a second MCU - ESP8266, which implements the WiFi connectivity. This MCU needs separate firmware and communicates with the STM32 via an SPI port. The pin header next to the ESP module provides direct access to the programming pins for fast firmware flashing. At this stage, we experiment with the open-source firmware ESPLink [21]. It is equipped with several important communication features - it implements a serial bridge, an MQTT client and it is capable of making REST requests to an Internet server, which makes possible the integration of the laser system in a wide range of newly built or existing communication infrastructures, e.g., [22]. The fast data transfer speed enables the use of the laser system in real-time monitoring applications such as the creation of a laser-based information screen in various industrial or public sectors using methods such as the one proposed in [23].

The serial bridge feature may be used if a fast low-level communication venue is needed, e.g., for a live animation feed from a smartphone or a PC. The MQTT client feature is well suited for receiving remote control commands, sending status updates and integrating the laser system into a functioning IoT infrastructure. The REST capabilities can be used for the direct communication with an Internet server - to send status updates and receive responses in the absence of an MQTT infrastructure. The advantage of using an open-source firmware for the ESP8266 $\mathrm{MCU}$ is that some important features for the WiFi-based communication are already implemented and we can use them without developing them ourselves.

There is a limited web server running on the ESP8266, which provides a browser-based Graphical User Interface (GUI). This GUI is used for parameter setting and helps with the remote debugging if something goes wrong with the main MCU. Some important parameters that are set through the GUI are the WiFi network mode - client or server, the WiFi SSID, password and authentication mode, the IP address and the communication port of the MQTT server, etc. The firmware supports DHCP and SNTP, which facilitates the IP address configuration and makes the current time available to the laser system.

The MQTT and REST functionalities allow us to focus only on the needed messages and status updates while sending and receiving them over a mature and well-tested low-level communication infrastructure. In the best case, a client will already have some existing IoT systems communicating over MQTT and the laser system will be integrated as one more IoT device for entertainment and marketing purposes. The ESP8266 MCU takes care of the communication details leaving only the creation and interpretation of the message contents to the main STM32 MCU. As the network communication typically comes hand in hand with a significant amount of development and execution overhead, having a secondary MCU running on an open-source firmware saves but development and run-time resources.

Last but not least, the remote debugging console accessible from the ESP8266 MCU facilitates the identification of problems that may occur within the STM32 
MCU. It enables support staff to login from a distance, look into status messages and issue service commands such as rebooting the main MCU. This feature may prove to be very convenient because it may save a trip to the customer premises if problems should happen. Even within a single building, it is often more convenient to monitor the laser system from the comfort of the working desk and chair instead of going with a notebook and connecting to it by wire.

We have dedicated a separate task in the STM32 MCU to communication. This task may receive commands to start, stop or change the animation; it may get new animation files over the $\mathrm{WiFi}$ connection and start a writing process to the microSD card; it may update the MCU firmware, send status updates, etc. As an alternative communication venue, it is also possible to establish a USB connection to a notebook or a PC using an add-on board with a serial-to-USB communication chip such as the FT232RL or the CP2102. The connection uses one of the five serial ports of the MCU. Programming the communication with the Internet as a separate task ensures the responsiveness of the system at any given point in time. Messages will be received and commands will be executed irrespectively of the other laser system tasks. As the MCU resources are shared, the execution may not be as fast as technologically possible but it will commence and proceed in a timely manner. In addition, by using separate tasks, we divide the firmware into relatively standalone and encapsulated parts facilitating the debugging and increasing the reliability - provided that the underlying FreeRTOS functions as intended.

Another normal-priority task controls the temperature and the humidity inside the laser system. As the system is intended to operate both outdoors and indoors, it has to monitor the temperature and the humidity values constantly because laser diodes should not be used at negative temperatures or at temperatures above $50{ }^{\circ} \mathrm{C}$. The humidity is a problem because of the possibility for condensation inside the camera. For this reason, the camera is equipped with a temperature sensor, a humidity sensor, a heating element and a fan. They are managed by a separate task that first measures the temperature and the humidity and then activates or deactivates the heating element and the fan.

The temperature sensor is encased in a standard Integrated Circuit (IC) housing mounted on the PCB. It is connected to one of the Analog-to-Digital Converter (ADC) channels of the $\mathrm{MCU}$ and it has a $2{ }^{\circ} \mathrm{C}$ measurement error, which is enough for our application. The humidity sensor is also an IC mounted on the PCB. It is controlled by the MCU over an Inter-Integrated Circuit (I2C) two-wire connection. The heating element consists of one or two aluminum resistors connected in series and mounted inside the enclosure. It is controlled by an MCU digital output via an N-MOS transistor, which is powerful enough to handle the necessary current. If the temperature falls below $5{ }^{\circ} \mathrm{C}$, the MCU turns the heating element on. The fan is similarly controlled via an N-MOS transistor. If the temperature rises above $40{ }^{\circ} \mathrm{C}$ or the humidity inside the enclosure is too high, the fan is turned on to cause air circulation and start normalizing the operating conditions. If for some reason this does not happen, the animation projection is halted until the environmental conditions are brought back to normal. In addition to the environmental parameters discussed above, the STM32 MCU has enough free inputs and outputs to gather data from other 
types of sensors - e.g., luminance sensors, movement sensors, etc., and to control actuators such as a Pan-Tilt-Zoom (PTZ) system or an alarm siren. This is considered for a future improvement of the smart compact laser system.

All tasks are performed in parallel so that no task remains active for too long. This guarantees the system responsiveness. Adding new tasks is relatively simple but it has to be considered that each task needs its own share of MCU resources. If need be, the MCU can be upgraded, e.g., to the STM32 F4 or F7 series, which are commonly used in robotics, e.g., [24], as they have more resources - e.g., execution speed of up to $216 \mathrm{MHz}$ and memory capacity of $256 \mathrm{~KB}$. This is possible because there is very good intercompatibility between the MCUs of the different STM32 Cortex-M series.

\section{Experimental results}

The initial tests show very promising results. The laser system successfully reads and projects laser animations with very good quality - Figs 5 and 6 . At the moment, we use only one color channel for test purposes as our color mix has to be tweaked by changing laser diodes due to the large difference in the power output between green diodes on one hand and red and blue diodes on the other hand. Larger laser systems use a string of laser diodes for each color channel with different number of diodes in each string for balancing the power output. In this system, due to size restrictions, we incorporate only one diode per color channel.

The most important point for us was to test whether the embedded DAC peripheral units of the MCU are fast enough to drive the laser scanners for an animation projection. As seen in the figures, the DAC speed is sufficient and even with cheap 20-kpps scanners the animation quality is very good.

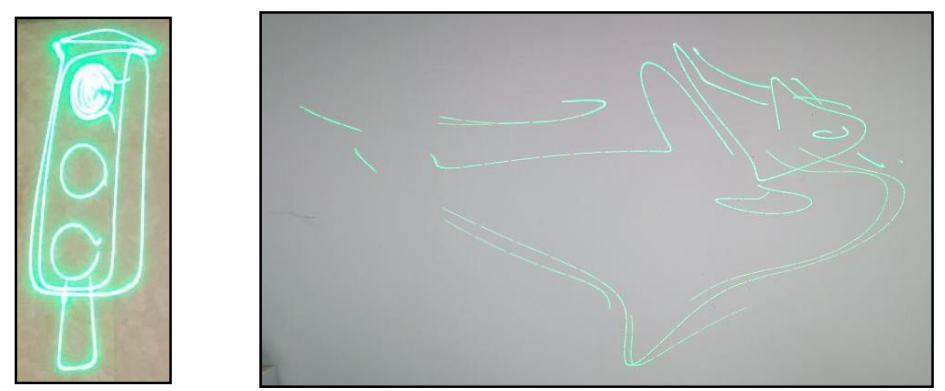

Fig. 5. Stylized laser animations projected by our system: traffic lights (left) and fish (right)

The experimental results also confirm that the ILDA animation files do not contain all parameters needed for a laser animation. For example, the laser beam blanking time has to be tweaked for each laser animation. For even better results, blanking time should be changeable for each animation frame transition. The improper setting of the blanking time leads to the gap in the circle in Fig. 6. In addition, the blanking time also depends on the scanners used in the laser system. In practice, this means that for each animation file and each laser system, there should be additional metadata containing information about the blanking time for all animation frame transitions. 

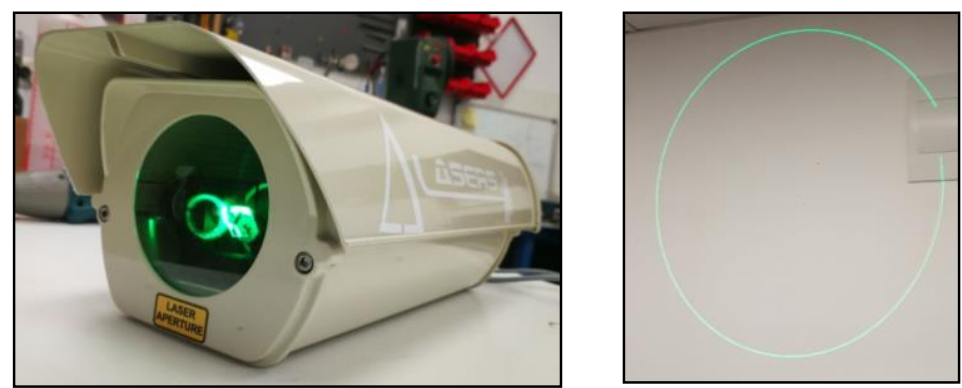

Fig. 6. The compact laser system (left) projecting a circle (right)

Another important point was to see if the MCU resources of an F3 series MCU are sufficient for the laser animation control, the real-time update of the memory double buffer, the communication with the Internet and the observation and control of the environmental parameters. The resources proved to be sufficient - for animations up to about 1500 laser points - which should be more than enough for most laser animations. The animations in Fig. 5 are relatively complex and contain respectively around 850 points (traffic lights) and 700 points (fish). They are projected fluently and without undue delays between the animation frames.

Should more resources be needed in the future, the MCU can be upgraded to the F4 or F7 series, which will require some adaptations of the software libraries but the hardware board and the main program code will remain unchanged. An MCU upgrade will allow us to raise the limit of 1500 animation points to a higher value. One should bear in mind though, that there is a practical limit of about 2000 points per animation frame that is imposed by the physical characteristics of the laser scanner units and cannot be alleviated so easily. A more powerful MCU may also allow us to perform some more complex actions such as on-board animation processing and special effects imposed over basic animation sequences.

The communication with the Internet could be setup successfully and with ease thanks to the ESP-link firmware. Still, it would be better if we had included in the design an on-board user panel with one or two navigation buttons and possibly a small LCD screen for immediate configuration feedback. As an alternative, the role of a status and configuration screen may also be played by the laser projection itself. With regard to navigation buttons, in the current prototype design, there is one general-purpose push-button mounted on the PCB inside the enclosure.

The environmental parameters are read successfully and the heating control works very well. Still, we need further tests outdoors and at negative temperatures to confirm the reliable animation projection in winter conditions.

\section{Conclusion}

In this paper, we propose and test a smart compact laser system for animation projections. We gather and summarize the most important user requirements and describe the design and implementation phases of the prototype development along with a brief discussion of the test results with suggestions for improvement of the created prototype. 
The proposed laser system has several advantages over existing laser systems. From a user perspective, the system is simple to use and easy to install and maintain due to its small dimensions and low weight. From a technical standpoint, our smart electronic control board integrates all necessary electronics with the exception of the scanner control and it enables the projection of laser animations in a standalone or a remote-controlled fashion. From an economic standpoint, we achieve lower cost than the purchase and maintenance cost of a typical multimedia laser system.

One limitation of the system is the absent ILDA interface for connection to popular laser show software - due to the limited enclosure dimensions and the specifics of the envisioned use cases. In the future, this limitation may be mitigated by the design of an optional add-on module that will provide the required analog support for processing ILDA input signals. Our future work will also include performing laser animation projections on the fly - by using data streamed over the network and animations that represent real physical objects or physical parameters. Such use cases may encompass the projection of the outlines of construction details for inspection or as assembly hints in factories or the real-time projection of manufacturing process parameters such as temperature, humidity or pressure. If we think of the laser system not only as an entertainment device but also as an innovative display or a virtual drawing board, new use cases may emerge in application fields such as industrial machinery, education and sports.

Acknowledgments: This research is supported by the Bulgarian FNI fund through the project "Conceptual Modeling and Simulation of Internet of Things Ecosystems (KoMEIN), contract ДН02/1 from 13.12.2016, and by the National Scientific Program "Information and Communication Technologies for a Single Digital Market in Science, Education and Security (ICTinSES)", financed by the Ministry of Education and Science, decree 203 of the Council of Ministers from 19.09.2018.

\section{References}

1. ILDA Technical Committee. The ILDA Standard Projector, July 1999 (Retreived on 29.07.2019). https://www.ilda.com/resources/StandardsDocs/ILDA_ISP99_rev002.pdf

2. Re d d in g, B., A. C erj a n, X. Hu an g, M. L. Le e, A. D. S ton e, M. A. Cho ma, H. C a o. Low Spatial Coherence Semiconductor Laser. - Proceedings of National Academy of Sciences, Vol. 112, February 2015, No 5, pp. 1304-1309. ISSN: 0027-8424, DOI: $10.1073 /$ pnas.1419672112.

3. F i s c h e r, F., G. P i 1 a r d. Laser Module Having a Duochromatic Laser Diode for a Portable Image Projector. 2016. US Patent US9485481B2.

http://patft.uspto.gov/netacgi/nph-

Parser?Sect2=PTO1\&Sect2=HITOFF \&p=1\&u=/netahtml/PTO/searchbool.html\&r=1\&f=G\&l=50\&d=PALL\&RefSrch=yes\&Query=PN/9485481

4. Th o m p s o n, B., S. Wurst er, L. A. Prather, D. S n ow, R. M c C a u l e y. Power Efficient Laser Diode Driver Circuit and Method. 2017. US Patent US9769459B2.

http://patft.uspto.gov/netacgi/nph-

Parser?Sect2=PTO1\&Sect2=HITOFF\&p=1\&u=/netahtml/PTO/searchbool.html\&r=1\&f=G\&l=50\&d=PALL\&RefSrch=yes\&Query=PN/9769459

5. W o l a k, E. Systems and methods to provide high brightness diode laser outputs. 2016. US Patent US9316846B2.

http://patft.uspto.gov/netacgi/nph-

Parser?Sect2=PTO1\&Sect2=HITOFF \&p=1\&u=/netahtml/PTO/searchbool.html\&r=1\&f=G\&l=50\&d=PALL\&RefSrch=yes\&Query=PN/9316846 
6. Li, F., J. Yablon, A. Velte n, M. Gupta, O. Cos s a irt. High-Depth-Resolution Range Imaging with Multiple-Wavelength Superheterodyne Interferometry Using 1550-nm Lasers. Applied Optics, Vol. 56, 2017, Issue 31, pp. H51-H56. Print ISSN: 1559-128X, Online ISSN: 2155-3165, https://doi.org/10.1364/AO.56.000H51

7. L i u, Q. et al. Charging Unplugged: Will Distributed Laser Charging for Mobile Wireless Power Transfer Work?. - IEEE Vehicular Technology Magazine, Vol. 11, 2016, No 4, pp. 36-45. ISSN: 1556-6072, DOI: 10.1109/MVT.2016.2594944.

8. D i n g, J., J. L i u, X. W e i, J. X u. The SMAT Fiber Laser for Industrial Applications. - In: Proc. of SPIE 10085, Components and Packaging for Laser Systems III, 100850R, 22 February 2017, ISSN: 0277-786X, DOI: 10.1117/12.2250459.

9. Chatzizyrli, E., N. Tinne, R. Lachmayer, J. Ne u mann, D. Kracht. Modeling of Photoluminescence in Laser-Based Lighting Systems. - In: Proc. of SPIE 10603, Photonics, Devices, and Systems VII, 1060318, 1 December 2017, ISSN: 0277-786X, DOI: $10.1117 / 12.2292735$. https://doi.org/10.1117/12.2292735

10. B a s u, C., M. M e i n h a r d t-W o 11 w e b e r, B. R o t h. Lighting with Laser Diodes. - Advanced Optical Technologies, Vol. 2, 2013, No 4, pp. 313-321. ISSN: 2192-8576.

11. Melville, C. D., R. S. Johnston, C. M. Lee, E. J. S e ibel, B. T. S chowengerdt. Scanning Laser Projection Display Devices and Methods for Projecting One or More Images onto a Surface with a Light-Scanning Optical Fiber. 2014. US Patent: US8757812B2.

12. Lutgen, S., D. Dini, I. Pietzonka, S. Tautz, A. Breidenasse 1, A. Le11, A. Avramescu, C. Eichler, T. Lermer, J. Müller, G. B ruederl, A. GomezI g l e si a s, U. S tra us s, W. G. S cheibenzuber, U. T. S c h warz, B. P a se n o w, S. K o c h. Recent Results of Blue and Green InGaN Laser Diodes for Laser Projection. - In: Proc. of SPIE 7953, Novel In-Plane Semiconductor Lasers X, 79530G, 16 February 2011, ISSN: 0277-786X, E-ISSN: 1996-756X, https://doi.org/10.1117/12.874757

13. B e 11 a n c o u r t, A. R., U. M a c k e n s, H. M o e n c h et al. Blue Diode Pumped Solid-State Lasers for Digital Projection. - Laser Physics, Vol. 20, 2010, Issue 3, pp. 643-648. ISSN: 1054-660X. https://doi.org/10.1134/S1054660X10050026

14. W e is s, M., D. Am y o t. Business Model Design and Evolution. Whitepaper. 2007. DOI: 10.1142/9789812770318_0013.

https://www.researchgate.net/publication/245591623_Business_Model_Design_and_Evo lution

15. A m y o t, D. Introduction to the User Requirements Notation: Learning by Example. - Computer Networks, Vol. 42, 2003, Issue 3, pp. 285-301. ISSN:1389-1286.

16. I l ch e v, S., Z. I l c h e v a. High-Speed Semiconductor Laser Diode Driver with Analog Signal Modulation. - In: International Conference "Big Data, Knowledge and Control Systems Engineering” (BdKCSE’2018), 21 November 2018, pp. 81-90. Publisher: John Atanasoff Society of Automatics and Informatics, ISSN: 2367-6450.

17. P e t k o v, D. RGB Laser Projector Systems. (Retreived on 02.08.2019). http://www.deltalasers.com

18. I 1 c h e v, S., R. A n d r e e v, Z. I l c h e va. HybridNET Management and Sensor Data Acquisition System. - In: IoT 2017: 7th International Conference on the Internet of Things, Linz, Austria, October 22-25, 2017, pp. 32:1-32:2, ACM Digital Library, ISBN: 978-1-4503-5318-2, DOI: $10.1145 / 3131542.3140268$.

19. I $1 \mathrm{ch}$ e v, S., Z. I $1 \mathrm{ch}$ e va. Internet-of-Things Communication Protocol for Low-Cost Devices in Heterogeneous Wireless Networks. - In: 18th International Conference on Computer Systems and Technologies (CompSysTech'17), Ruse, Bulgaria, ACM ICPS, Vol. 1369, 23-24 June 2017, pp. 272-279. ISBN: 978-1-4503-5234-5, DOI: 10.1145/3134302.3134329.

20. ILDA Technical Committee. ILDA Image Data Transfer Format Specification, 16 November 2014. (Retreived on 29.07.2019). https://www.ilda.com/resources/StandardsDocs/ILDA_IDTF14_rev011.pdf

21. W i p p le r, J . C. ESP-Link ((Retreived on 29.07.2019). https://github.com/jeelabs/esp-link 
22. A t a n a s o v, I., A. N i k o lo v, E. P e n c h e v a. Reducing Energy Consumption by Using Smart Metering Intelligent Systems. - Cybernetics and Information Technologies, Vol. 16, 2016, No 2, pp. 113-124.

23. M a, Z., W. Li u. Data Receiving Method Based on Multimedia Timing in Real-Time System. Cybernetics and Information Technologies, Vol. 17, 2017, No 1, pp. 126-134.

24. C a i, J., X. Z h o u, Z. P a n, P. G a o, Y. L u o, Z. L i n. Study on the Design and Control of Pipeline Leak Detection Robot Fish. - Cybernetics and Information Technologies, Vol. 18, 2018, No 3, pp. 120-131.

Received: 27.06.2019; Second Version:12.08.2019; Accepted: 22.08.2019 